\title{
Optical Phase Transition in Semiconductor Quantum Metamaterials
}

\author{
Adrian Hierro, ${ }^{1, *}$ Miguel Montes Bajo, ${ }^{1, *}$ Mario Ferraro, ${ }^{2,}{ }^{*}$ Julen Tamayo-Arriola,${ }^{1}$ Nolwenn Le Biavan, ${ }^{2}$ Maxime Hugues,${ }^{2}$ \\ Jose M. Ulloa, ${ }^{1}$ Massimo Giudici, ${ }^{3}$ Jean-Michel Chauveau, ${ }^{2, \dagger}$ and Patrice Genevet ${ }^{2, *}$ \\ ${ }^{1}$ ISOM, Universidad Politécnica de Madrid, Avenida Complutense 30, 28040 Madrid, Spain \\ ${ }^{2}$ Université Cote d'Azur, CNRS, CRHEA, rue Bernard Gregory, Sophia Antipolis, 06560 Valbonne, France \\ ${ }^{3}$ Université Cote d'Azur, CNRS, InPhyni, Route des Lucioles, Sophia Antipolis, 06560 Valbonne, France
}

(Received 23 January 2019; published 10 September 2019)

\begin{abstract}
Unexpected light propagation effects, such as negative refraction, have been reported in artificial media. Leveraging on the intersubband resonances in heterostructured semiconductors, we show that all possible optical regimes, ranging from classical dieletric and metal to hyperbolic metamaterial types 1 and 2 , can be achieved. As a demonstration, we prove that the negative refraction effect can occur at a designed frequency by controlling the electronic quantum confinement.
\end{abstract}

DOI: 10.1103/PhysRevLett.123.117401

Metamaterials are artificial, human designed materials that feature uncommon physical properties such as an ultrahigh refractive index, extraordinary optical activity, and generalized reflection and refraction [1-5]. In modern optics, these metamaterials are becoming ubiquitous, in particular owing to their fascinating possibility of realizing devices based on a negative index of refraction. Originally, materials with negative refraction index were theoretically considered by Veselago in 1968 [6]. The negative index is obtained in a material presenting simultaneous negative dielectric permittivity and magnetic permeability. In Veselago's method, metamaterials are designed by matching electric and magnetic resonances, leading to a strong absorption. Since then, much effort has been made to reduce this absorption, considering, for example, gain materials [7,8]. Another efficient approach consists of exploiting the hyperbolic dispersion of light propagating in materials featuring opposite signs of permittivity along the orthogonal in- and out-of-plane directions [9]. Hyperbolic dispersion is reached in an anisotropic medium by modifying the optical response along one direction, using, for example, an arrangement of materials of opposite sign of permittivities. In such a system, also dubbed a hyperbolic metamaterial (HMM), one of the electric or magnetic resonances is replaced by the material anisotropy. HMMs are of type 1 or type 2, whether they possess one or two negative permittivity components. Type $1 \mathrm{HMMs}$ are more suitable for practical applications thanks to the propagation effects inside the metamaterial. They exhibit a negative refraction without negative index due to the opposite signs of the in-plane components of Poynting and wave vectors. On the other hand, type 2 HMMs reflect all incident light and do not show negative refraction $[10,11]$. This conceptual simplification comes with a drastic reduction of the optical losses since the main idea is to use an alternating composition of resonant and nonresonant materials. Semiconductor materials offer several functional advantages for HMMs by substituting the metallic resonant part with a highly doped semiconductor [12-14]. Unfortunately, one of the most important features of a semiconductor heterostructure has been disregarded so far, i.e., the quantum confinement and the related electronic transitions.

In this Letter, we address the fundamental role of quantum heterostructures inserted into a HMM. Contrary to the previous works on semiconductor HMMs, our contribution accounts not only for the in-plane metallic response but also for the presence of strong electronic resonances along the $z$ direction due to the intersubband (ISB) transitions in quantum wells (QWs). Tuning both the QW thickness and the barrier height allows us to tune the optical phase diagram, ranging from classical dielectric and metal to type 1 and 2 HMM.

Before getting into the details of the metamaterial designs, we propose an intuitive toy model that contains all of the physical ingredients leading to HMM regimes. Let us consider a stack composed of two alternating materials: (1) a nondispersive dielectric, and (2) a layer exhibiting a resonant Lorentzian response [see Fig. 1(a)]. The resonant medium is represented by an oscillating charge on a spring. In the hyperbolic regime, the thicknesses of both layersnamely, $l_{1}$ and $l_{2}$-are subwavelength, and, thus, the permittivity of the whole structure can be described using the Maxwell-Garnett effective-medium theory. Then the effective permittivity of the structure can be averaged from the layer medium permittivities ( $\varepsilon_{1}$ and $\varepsilon_{2}$, respectively) as follows:

$$
\begin{gathered}
\varepsilon_{\text {in-plane }}=\frac{1_{1} \varepsilon_{1}+1_{2} \varepsilon_{2}}{l_{1}+l_{2}}, \\
\varepsilon_{z}=\left(\frac{\frac{1_{1}}{\varepsilon_{1}}+\frac{l_{2}}{\varepsilon_{2}}}{l_{1}+l_{2}}\right)^{-1} .
\end{gathered}
$$


Keeping $\varepsilon_{2}$ constant, a sign inversion of $\varepsilon_{1}$ is required to achieve opposite signs of the in- and out-of-plane permittivities. A Lorentzian susceptibility can become negative by introducing any physical phenomenon that can be described as a harmonic oscillator mode such as dipole excitations, twolevel quantum transitions or other effects (see the Supplemental Material [15]). Taking $\omega_{0}$ to be the resonance frequency of medium 1 and $\gamma$ its broadening, we can write

$$
\varepsilon_{1}(\omega)=\varepsilon_{\infty}+\frac{\omega_{p}^{2}}{\omega_{0}^{2}-\omega^{2}-i \omega \gamma}
$$

where $\omega_{p}$ represents the collective oscillation frequency of the ensemble of springs in medium 1 and $\varepsilon_{\infty}$ its highfrequency permittivity. The optical phase diagram is obtained by substituting Eq. (3) into Eqs. (1) and (2). It exhibits a phase transition from dielectric to metal through type 1 and 2 hyperbolic metamaterials, as shown in Fig. 1(b). The sign inversion conditions of the real part of the permittivity are highlighted for both components in the figure. An intersection between these two curves occurs, giving rise to a hyperbolic zone. The in-plane resonance occurs at $\omega=\omega_{0}$. Along the $z$ axis, it occurs at

$$
\omega=\sqrt{\omega_{0}^{2}+\frac{\omega_{p}^{2}}{\varepsilon_{\infty}} .}
$$

It represents the maximum excitation frequency to achieve a hyperbolic behavior for negligible absorption $\left(\gamma \ll \omega_{p}\right)$. Negative index of refraction occurs when the composite material reaches type 1 hyperbolic dispersion [12,16-18]. In such a material, an incoming light beam is refracted at negative angle, i.e., in the opposite direction with respect to the refracted beam at the interfaces with conventional materials, as presented in Fig. 1(c).

Such HMM behavior can be experimentally realized by relying on ISB transitions in QWs. Let us consider a multiple quantum well (MQW) with highly doped QWs and undoped barriers with a spatial period $\Gamma \ll \lambda$, where $\lambda$ is the wavelength of light. Because of the confinement in the $z$ direction, the electrons can oscillate between two discrete electronic states at a frequency $\omega_{0}=\omega_{12}$, behaving as a two-level quantum system. The oscillator frequency $\omega_{12}$ and, in turn, the Lorentzian resonance frequency in Eq. (3) is tuned by the QW thickness and barrier height. Owing to the selection rules, the intersubband transition is dipole forbidden in the in-plane direction $\left(\omega_{12}^{\text {in-plane }}=0\right)$, leading to an anisotropic permittivity [18].

To demonstrate this novel approach, we rely on ISB transitions in (1010) $\mathrm{ZnO} /(\mathrm{Zn}, \mathrm{Mg}) \mathrm{O}$ MQWs [19]. High quality nonpolar $\mathrm{ZnO}$ heterostructures can be grown on bulk $\mathrm{ZnO}$ substrates at high doping concentration (well above $10^{19} \mathrm{~cm}^{-3}$ ) [20-22], which facilitates the control of the ISB transitions by avoiding the quantum confined Stark effect. The structures were grown by molecular beam

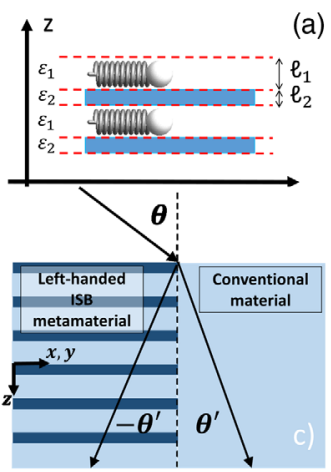

(a)

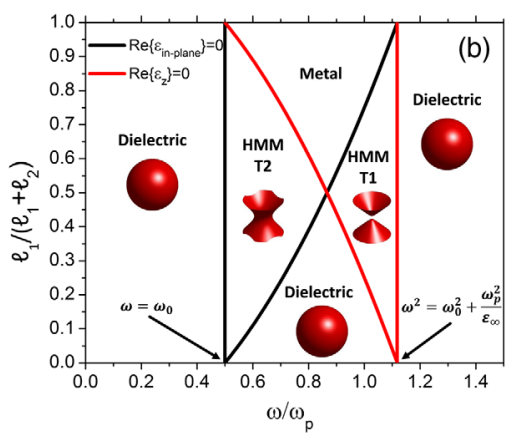

FIG. 1. (a) Schematic of the toy model structure. A stack along the $z$ direction of two alternating media: layer 1, thickness $l_{1}$ and a resonant permittivity $\varepsilon_{1}$; layer 2 , thickness $l_{2}$, dispersionless with a permittivity $\varepsilon_{2}$. (b) Optical phase diagram showing the sign inversion conditions for both in-plane and $z$ components of the effective permittivity as a function of the normalized frequency $\omega / \omega_{p}$ and of the thickness ratio. 3D inset images: isofrequency surfaces plotted in $k$ space for the different behaviors. The resonance energy is $\omega_{0}=\omega_{p} / 2$. For simplicity, the curves are plotted imposing $\varepsilon_{2}=\varepsilon_{\infty}=1$ and $\gamma=0$. (c) Comparison between the refraction effect in (right) a conventional and in (left) a negative index material.

epitaxy (MBE) on $m$-plane $\mathrm{ZnO}$ substrates. They consist of a ten-period $\mathrm{ZnO} / \mathrm{Mg}_{0.35} \mathrm{Zn}_{0.65} \mathrm{O} \mathrm{MQW}$. The $\mathrm{ZnO}$ QWs are $n$ doped $\left([\mathrm{Ga}] \sim 5 \times 10^{19} \mathrm{~cm}^{-3}\right)$, and the $\mathrm{Mg}_{0.35} \mathrm{Zn}_{0.65} \mathrm{O}$ barriers are nonintentionally doped. The doping concentration is high enough for the in-plane susceptibility to be metallic. Since the barrier is undoped, it is considered to be an isotropic dielectric material. Note that the utilization of the MBE technique to grow the HMM structures allows the reduction of the surface roughness and improves the material quality of the HMM stack.

In order to model the response of a $\mathrm{ZnO} /(\mathrm{Zn}, \mathrm{Mg}) \mathrm{O}$ multilayer in the mid-IR, a permittivity that accounts for the interaction of light with both the lattice vibrations and the electron plasma [23] should be considered. Here, the ISB resonance is chosen to be far from the phonon modes $\left(\omega_{\text {phonons }} \leq 675 \mathrm{~cm}^{-1}\right)$. Thus the influence of the phonon modes can be neglected. The dielectric permittivity of the $(\mathrm{Zn}, \mathrm{Mg}) \mathrm{O}$ barrier is nearly isotropic and can be expressed by $\varepsilon^{\mathrm{MgZnO}}=\varepsilon_{\infty}^{\mathrm{MgZnO}}$, where $\varepsilon_{\infty}^{\mathrm{MgZnO}}$ is the high-frequency dielectric constant. In the doped $\mathrm{ZnO}$ QWs, the isotropy is not preserved anymore due to the presence of the electronic transitions. More specifically, the high doping concentration in the $\mathrm{ZnO}$ layers results in the presence of a quasi-two-dimensional electron gas in the $x-y$ plane, behaving as a metallic thin film described by a Drude term in the permittivity as

$$
\varepsilon_{\text {in-plane }}^{\mathrm{ZnO}}(\omega)=\varepsilon_{\infty}^{\mathrm{ZnO}}-\frac{\omega_{P}^{2}}{\omega^{2}+i \omega \gamma_{\text {in-plane }}^{P}} .
$$


The parameter $\gamma_{\text {in-plane }}^{P}$ stands for the broadening of the plasma resonance at frequency $\omega_{p}$ given by

$$
\omega_{P}^{2}=\frac{n_{2 \mathrm{D}} e^{2}}{m^{*} L_{\mathrm{QW}} \varepsilon_{0}}
$$

where $n_{2 \mathrm{D}}$ is the two-dimensional electron gas concentration in the $\mathrm{QW}, \varepsilon_{0}$ is the permittivity of vacuum, $e$ is the electron charge, $m^{*}$ is the electron effective mass, and $L_{\mathrm{QW}}$ is the quantum well width.

Along the growth direction, the optical response must take into account the ISB transition. This effect is well captured by considering an oscillation of the electrons between the two sublevels in the conduction band $[18,24,25]$. This contribution can be modeled by considering a Lorentzian term in the permittivity, as

$$
\varepsilon_{z}^{\mathrm{ZnO}}(\omega)=\varepsilon_{\infty}^{\mathrm{ZnO}}+\frac{f_{12} \omega_{P}^{2}}{\omega_{12}^{2}-\omega^{2}-i \omega \gamma_{12}},
$$

where $f_{12}$ is the ISB transition oscillator strength, $\omega_{12}$ the ISB transition frequency, and $\gamma_{12}$ its broadening.

The effect induced by the ISB transition on the permittivity is hence anisotropic (see the Supplemental Material [15]). While the in-plane component of the permittivity is unaffected by the presence of the ISB resonance, the presence of an ISB transition breaks the symmetry of the bulk optical response in the $z$ direction, which can be tuned by the layer thickness. As the QW width is increased, the number of sublevels in the QW increases, and ISB transitions between different levels could occur. Nevertheless, in the presence of a high doping level, many body interaction effects induce a coherence in the system, leading to a single strong dipole resonance, in which all dipoles in the different transitions are coupled and oscillate resonantly in phase. A mechanical analogue is the motion of the center of mass of an array of particles attached to each other by springs and oscillating in phase. The effective single resonance arising from the mode locking of ISB dipoles that contribute by their oscillation strengths is dubbed the multisubband plasmon (MSP) mode [26,27]. Since the MSP mode can be represented by an oscillation between two virtual confined levels in the QW, the single Lorentzian resonance model with the permittivity function in Eq. (7) is still valid. However, the mode frequency $\left(\omega_{12}\right)$ and the plasma frequency $\left(\omega_{p}\right)$ need to be adjusted as a function of the individual ISB transitions to capture correctly the MSP response [23]. A high doping level in the QWs leads to an additional shift of the ISB absorption peak $\left(\omega_{\text {abs }}\right)$ such that $\omega_{\text {abs }}^{2} \cong \omega_{12}^{2}+\omega_{p}^{2} / \varepsilon_{\infty}$ [28]. This effect is known as the depolarization shift, and it results from the screening of the electric field by the oscillating electrons in the system. Therefore, the final ISB absorption from the QW is a function not only of the energy difference between sublevels but also of the electron concentration in the layer.

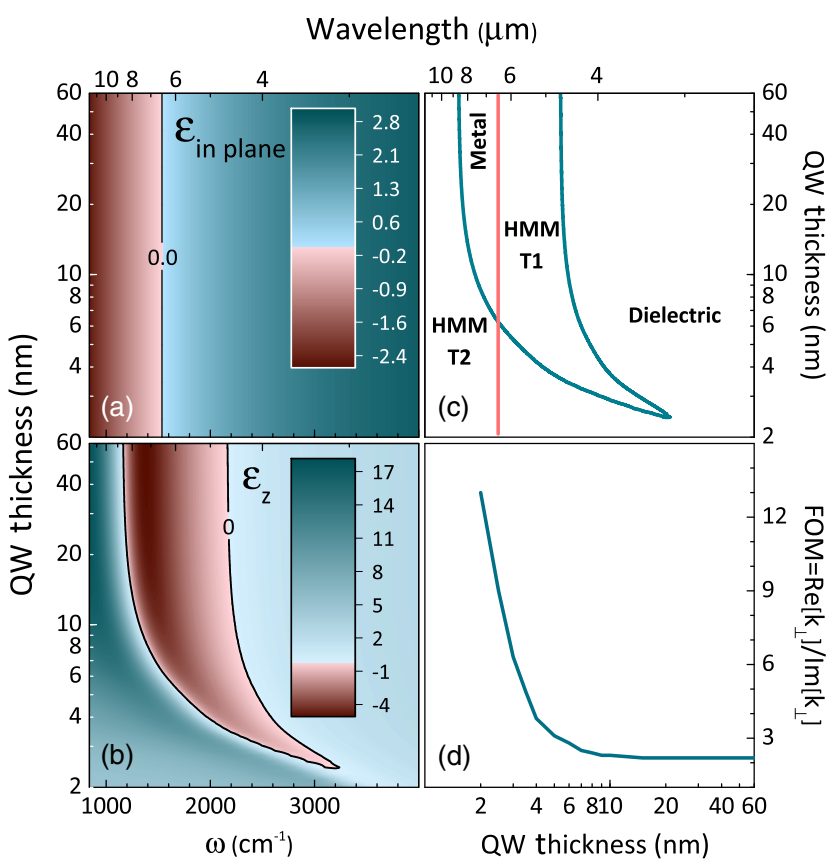

FIG. 2. Hyperbolic metamaterial condition with a nominal doping of $n \sim 5 \times 10^{19} \mathrm{~cm}^{-3}$ and $l_{\mathrm{ZnO}} / l_{\mathrm{MgZnO}}=3.5$. (a),(b) Real part of the effective permittivity (a) in-plane and (b) out-of-plane as a function of the frequency and QW thickness. The black solid curves show the sign inversions. (c) Optical phase transition diagram of the system as a function of frequency and QW thickness. (d) $\mathrm{FOM}=\operatorname{Re}\left(k_{\perp}\right) / \operatorname{Im}\left(k_{\perp}\right)$, where $k_{\perp}$ is the wave vector in the direction perpendicular to the layers.

A comparison of this expression with Eq. (4) for a metamaterial where $\operatorname{Re}\left\{\varepsilon_{z}(\omega)\right\}=0$ [see Fig. 1(b)] indicates that the maximum frequency at which the HMM is type 1 appears at the ISB absorption peak frequency $\left(\omega_{\mathrm{abs}}\right)$.

Figure 2 displays the spectral dependence of the permittivities for a MQW as a function of the QW thickness calculated by substituting Eqs. (5) and (7) into Eqs. (1) and (2). Both in- and out-of-plane effective permittivities exhibit a sign inversion, given by the curves $\operatorname{Re}\left\{\varepsilon_{\text {in-plane }}\left(l_{\mathrm{ZnO}}, \omega\right)\right\}=0$ and $\operatorname{Re}\left\{\varepsilon_{z}\left(l_{\mathrm{ZnO}}, \omega\right)\right\}=0$, underlined by the solid lines in Figs. 2(a) and 2(b). These two solid curves are plotted together in the diagram in Fig. 2(c) to illustrate the optical phase transition occurring in this system as a function of the QW thickness and wave number. By changing the QW thickness, i.e., $\omega_{12}$, we can obtain all of the possible optical behaviors, ranging from classical dielectric and metal to HMM type 1 and 2. The optical phase diagram also shows a singular point where the four optical behaviors meet and where the transition between the optical phases becomes highly sensitive to the system parameters. Remarkably, changing the ISB transition energy allows us to tune the HMM type 1 optical response by around $50 \%$ of the bulk plasma resonance, i.e., by around $1000 \mathrm{~cm}^{-1}$, just by modifying the QW thickness. As opposed to bulk materials without 
ISB transitions (i.e., with $\omega_{12}=0$ ), the optical phases can be modified here without changing the plasma frequency of the system. Tuning the resonance energy is a unique characteristic of this type of quantum metamaterial. Remarkably, the quantum confinement enhances the negative refraction effect in a type $1 \mathrm{HMM}$. This effect can be quantified with a figure of merit (FOM) given by FOM = $\operatorname{Re}\left(k_{\perp}\right) / \operatorname{Im}\left(k_{\perp}\right)$ (see the Supplemental Material [15] and Ref. [29]), which reaches values above 12 [Fig. 2(d)], comparing well with the state of the art $[11,13]$. Hence, we demonstrate that quantum confinement increases strongly the FOM compared to the negative index of refraction metamaterials in the classical limit (i.e., with no quantum confinement). Finally, note that, for large QW widths, the out-of-plane permittivity converges to its bulk material value. In this limit, the MSP mode frequency tends to zero, and its plasma frequency to the bulk plasma frequency of the $\mathrm{ZnO}$ layer.

The optical phase diagram of this system can also be controlled by adjusting the barrier height, which impacts $\omega_{12}$. In our material system, this parameter is determined by the $\mathrm{Mg}$ concentration in the barrier. Performing the equivalent analysis to Fig. 2, we show that varying the $\mathrm{Mg}$ concentration in the ( $\mathrm{Zn}, \mathrm{Mg}) \mathrm{O}$ barrier from $0 \%$ to $40 \%$ can tune the HMM type 1 behavior by up to $780 \mathrm{~cm}^{-1}$ (see the Supplemental Material [15]).

To test the validity of our theoretical model, we have fabricated a MQW structure with a designed depolarizationshifted ISB resonance at $\omega_{\text {abs }} \approx 2150 \mathrm{~cm}^{-1}$ [Fig. 3(a)]. The thicknesses were set at 35 and $10 \mathrm{~nm}$ for the QWs and the barriers, respectively. From polarization reflectance spectroscopy, we have extracted the material parameters and constructed the effective-medium permittivity (see the Supplemental Material [15]). As shown in Fig. 3(b), the MQW fulfills the requirements for a type $1 \mathrm{HMM}$ $\left(\varepsilon_{\text {in-plane }}>0\right.$ and $\left.\varepsilon_{z}<0\right)$ in the range from $\sim 1600$ to $2150 \mathrm{~cm}^{-1}$, which is also precisely the range in which the ISB transition is experimentally observed [Fig. 3(a)]. In agreement with the previously discussed model, the ISB transition is centered at the frequency where the out-ofplane permittivity crosses zero. Figures 3(c) and 3(d) show the relative transmittance (ratio of half-blocked to unblocked transmittance spectra; see the methods section in the Supplemental Material [15]) measured under $p$ and $s$ polarization, respectively, as a function of the angle of incidence on the HMM. The negative refraction is expected to give rise to a dip or an increase in the relative transmittance spectra. The relative transmittance measured under $p$ polarization [Fig. 3(c)] features a strong variation of the transmission across the frequency band centered at $2150 \mathrm{~cm}^{-1}$ (i.e., around $\omega_{\text {abs}}$ ). As predicted by the model, this effect is not present when repeating the experiment under $s$ polarization of the light [Fig. 3(d)]. Moreover, the sign and the intensity of the effect matches the prediction: for negative angles of incidence, the relative transmission

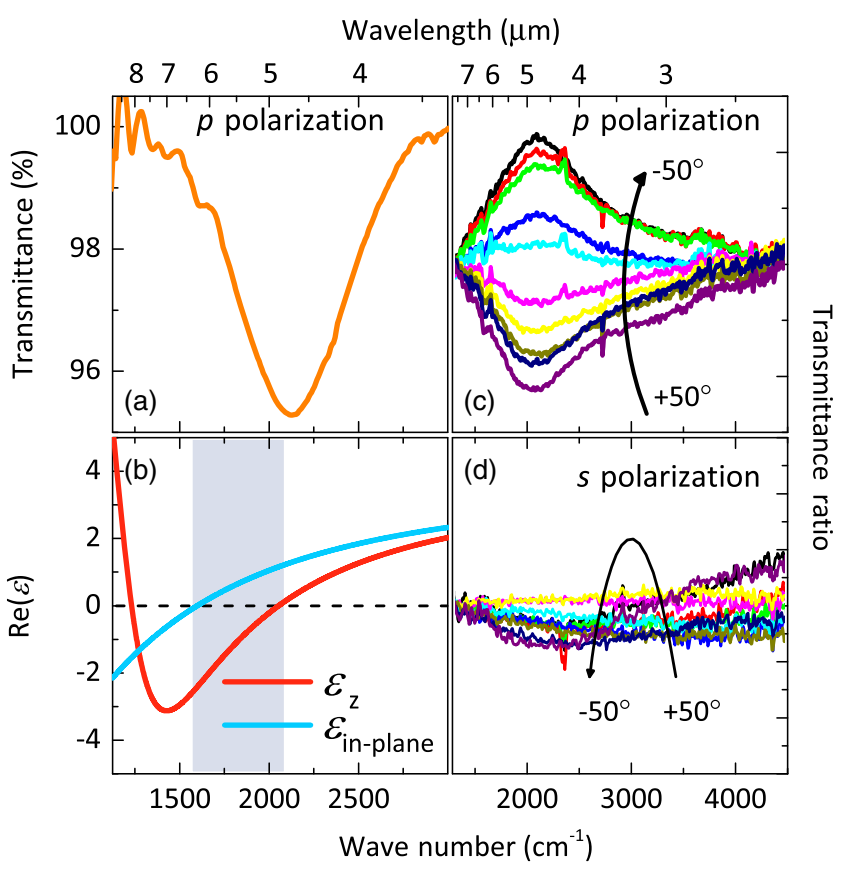

FIG. 3. (a) MQW measured transmittance under $p$ polarization at a $50^{\circ}$ angle of incidence, indicating the absorption at the ISB resonance. (b) Calculated real part of the in-plane and out-ofplane components of the effective permittivity of the sample. The shaded area indicates the region where the structure fulfills the requirements of a type $1 \mathrm{HMM}$. Ratio of the half-blocked to the unblocked transmittance spectra for (c) $p$ and (d) $s$ polarizations of the incoming light. The spectra at normal incidence have been taken as a baseline and subtracted from all other spectra. The arrows indicate the overall evolution of the spectra with the angle of incidence of the light.

increases with decreasing angles, whereas for positive angles it decreases with increasing angle. This result is a direct indication that the MQW structure features negative index of refraction at the frequency of the ISB resonance. Also, note that the observed increase in the relative transmittance for the negative incident angles indicates that we are observing negative index of refraction and not just absorption from the ISB transition.

In conclusion, we have predicted and experimentally demonstrated that the quantized electronic transitions in a quantum semiconductor system lead to hyperbolic behavior. The out-of-plane contribution of the intersubband transition in the QW permittivity enables a type 1 hyperbolic dispersion responsible for the negative refraction. This Letter connects for the first time the concepts of intersubband plasmons with the photonic response of hyperbolic metamaterials. Both the quantum well and barrier thicknesses and their respective doping are determinant for controlling the electronic resonance used to finely tune the material dispersion. This Letter highlights the role of quantum confinement in the photonic response of quantum heterostructure materials, and it proves that the electronic intersubband resonance in some of the most 
conventional heterostructured materials could lead to unexpected metamaterial behavior such as optical phase transitions. These results could be used to further control the photonic response and the dispersion properties of various quantum devices using intersubband transitions such as quantum cascade lasers and quantum well infrared detectors. Moreover, it has been shown that the large effective refractive index modification induced by the HMM response can be utilized to fabricate hyperbolic nanoantennas to control both scattering and absorption cross sections over a wide wavelength range [30].

The authors would like to thank Professor Vasanelli and Professor Vinter for the very helpful discussions and suggestions regarding the modeling part of the multisubband plasmon resonance. Part of the calculations performed here used the AESTIMO solver [31]. This work has been founded by the European Union's Horizon 2020 under the European Research Council (ERC) Grant Agreement No. 639109 (project Flatlight) and the Research and Innovation Program under Grant Agreement No. 665107 (project ZOTERAC). P. G., M. G., and M. F. acknowledges financial support from the Provence Alpes Côte d'Azur region. J. T.-A. holds a Predoctoral Contract from Universidad Politécnica de Madrid. A. H. acknowledges financial support from the Spanish Ministry of Economy and Competitiveness (MINECO) through Project No. TEC2017-85912-C2-1-R.

*These authors contributed equally to this work.

Corresponding author.

jmc@crhea.cnrs.fr

*Corresponding author.

Patrice.Genevet@crhea.cnrs.fr

[1] M. W. Klein, Science 313, 502 (2006).

[2] S. Zhang, Y.-S. Park, J. Li, X. Lu, W. Zhang, and X. Zhang, Phys. Rev. Lett. 102, 023901 (2009).

[3] A. K. Popov, S. A. Myslivets, and V. M. Shalaev, Opt. Lett. 34, 1165 (2009).

[4] D. R. Smith, P. Kolinko, and D. Schurig, J. Opt. Soc. Am. B 21, 1032 (2004).

[5] N. Yu, P. Genevet, M. A. Kats, F. Aieta, J. P. Tetienne, F. Capasso, and Z. Gaburro, Science 334, 333 (2011).

[6] V. G. Veselago, Sov. Phys. Usp. 10, 509 (1968).

[7] A. Fang, T. Koschny, M. Wegener, and C. M. Soukoulis, Phys. Rev. B 79, 245127 (2009).

[8] A. De Luca, M. P. Grzelczak, I. Pastoriza-Santos, L. M. Liz-MarzáN, M. La Deda, M. Striccoli, and G. Strangi, ACS Nano 5, 5823 (2011).
[9] A. Poddubny, I. Iorsh, P. Belov, and Y. Kivshar, Nat. Photonics 7, 948 (2013).

[10] A. J. Hoffman, L. Alekseyev, S. S. Howard, K. J. Franz, D. Wasserman, V. A. Podolskiy, E. E. Narimanov, D. L. Sivco, and C. Gmachl, Nat. Mater. 6, 946 (2007).

[11] D. Korobkin, B. Neuner, C. Fietz, N. Jegenyes, G. Ferro, and G. Shvets, Opt. Express 18, 22734 (2010).

[12] G. V. Naik, J. Kim, and A. Boltasseva, Opt. Mater. Express 1, 1090 (2011).

[13] A. J. Hoffman et al., J. Appl. Phys. 105, 122411 (2009).

[14] G. V. Naik, J. Liu, A. V. Kildishev, V. M. Shalaev, and A. Boltasseva, Proc. Natl. Acad. Sci. U.S.A. 109, 8834 (2012).

[15] See Supplemental Material at http://link.aps.org/ supplemental/10.1103/PhysRevLett.123.117401 for a detailed description of the calculations leading to the formula used in the paper and experimental methods.

[16] V. A. Podolskiy and E. E. Narimanov, Phys. Rev. B 71, 201101 (2005).

[17] K. V. Sreekanth, A. De Luca, and G. Strangi, Appl. Phys. Lett. 103, 023107 (2013).

[18] M. Helm, in Intersubband Transitions in Quantum Wells: Physics, and Device Applications, edited by H. C. Liu and F. Capasso (Academic Press, New York, 1999).

[19] N. Le Biavan et al., Appl. Phys. Lett. 111, 231903 (2017).

[20] J. M. Chauveau, M. Teisseire, H. Kim-Chauveau, C. Deparis, C. Morhain, and B. Vinter, Appl. Phys. Lett. 97, 081903 (2010).

[21] S. Brochen, G. Feuillet, J.-L. Santailler, R. Obrecht, M. Lafossas, P. Ferret, J.-M. Chauveau, and J. Pernot, J. Appl. Phys. 121, 095704 (2017).

[22] S. Kalusniak, S. Sadofev, and F. Henneberger, Phys. Rev. Lett. 112, 137401 (2014).

[23] M. Montes Bajo, J. Tamayo-Arriola, M. Hugues, J. M. Ulloa, N. Le Biavan, R. Peretti, F. H. Julien, J. Faist, J.-M. Chauveau, and A. Hierro, Phys. Rev. Applied 10, 024005 (2018).

[24] P. Yu and M. Cardona,Fundamentals of Semiconductors: Physics and Materials Properties (Springer, Berlin, 2010).

[25] W. P. Chen, Y. J. Chen, and E. Burstein, Surf. Sci. 58, 263 (1976).

[26] G. Fasol, N. Mestres, H. P. Hughes, A. Fischer, and K. Ploog, Phys. Rev. Lett. 56, 2517 (1986).

[27] A. Delteil, A. Vasanelli, Y. Todorov, C. Feuillet Palma, M. Renaudat St-Jean, G. Beaudoin, I. Sagnes, and C. Sirtori, Phys. Rev. Lett. 109, 246808 (2012).

[28] S. J. Allen, D. C. Tsui, and B. Vinter, Solid State Commun. 20, 425 (1976).

[29] V. M. Shalaev, Nat. Photonics 1, 41 (2007).

[30] N. Maccaferri, Y. Zhao, T. Isoniemi, M. Iarossi, A. Parracino, G. Strangi, and F. De Angelis, Nano Lett. 19, 1851 (2019).

[31] See http://www.aestimosolver.org/. 